\title{
SLEEP DURATION AND METABOLIC SYNDROME IN OBESE ADOLESCENTS
}

\author{
Nur Aisyah Widjaja ${ }^{1 *}$, Rendi Aji Prihaningtyas ${ }^{1}$, Roedi $\operatorname{Irawan}^{1}$, Meta Herdiana Hanindita ${ }^{1}$ \\ ${ }^{1}$ Child Health Department, Faculty of Medicine, Dr Soetomo General Hospital, Surabaya, Indonesia \\ *E-mail:nuril08@yahoo.com
}

\begin{abstract}
Shorter sleep duration is a risk factor for obesity and metabolic syndrome. Previous studies conducted on different races showed inconsistent results. The purpose of this study was to analyze the differences in sleep duration in obese adolescents who suffer from metabolic syndrome compared with obese adolescents who do not suffer from metabolic syndrome. A cross sectional study was carried out on 59 obese adolescents who visited the Pediatric Nutrition and Metabolic Disease Clinic in Dr. Soetomo General Academic Hospital, Surabaya. Subjects were selected using total sampling techniques who met the inclusion and exclusion criteria in August-November 2018. Anthropometry (weight, height and waist circumference), blood pressure, and blood tests (HDL cholesterol levels, triglycerides, and fasting blood glucose levels) were held to determine obesity according to CDC 2000 and metabolic syndrome according to International Diabetes Federation. The difference in sleep duration in obese adolescents suffering from metabolic syndrome and without metabolic syndrome analyzed using Chi square test. A total of 27 subjects (45.8\%) suffered from metabolic syndrome. Most obese adolescents (57,6\%) have sufficient sleep duration ( $\geq 8$ hours/day). There was no sleep duration differences in obese adolescents suffering and not suffering from metabolic syndrome $(p>0.05)$.
\end{abstract}

Keywords: Obesity, Sleep Duration, Metabolic Syndrome, Adolescents

\section{INTRODUCTION}

Obesity is a global health problem today in all ages. The incidence of obesity in adolescents is increasing and it causes metabolic syndrome. The incidence of obesity increases with the lack of sleep duration in adolescents (Seo \& Shim, 2019).

Metabolic syndrome is a collection of metabolic disorders consisting of several criteria based on the International Diabetes Federation (2007) including waist circumference $\geq 90$ percentiles for age and sex accompanied by 2 of the following criteria: (a) fasting triglyceride levels $\geq 150 \mathrm{mg} / \mathrm{dL}$, (b) fasting HDL cholesterol levels $<40 \mathrm{mg}$ / dL, (c) systolic or diastolic blood pressure $\geq 90$ percentile, and (d) fasting blood glucose levels $\geq 100 \mathrm{mg} / \mathrm{dL}$ (Zimmet, et al., 2007). Proinflammatory cytokines that are increased in obesity cause metabolic syndrome (Rochlani, et al., 2017). The prevalence of metabolic syndrome in obese adolescents is 5.5\% in males and 3.3\% in females (Seo \& Shim, 2019).

The duration of sleep for each individual is different, according to age. The sleep duration of a child is different from that of adolescents and adults. Adolescents aged 14-17 years are recommended to have sleep duration of $8-10$ hours/day. While sleep duration of 7-9 hours/day is recommended for adults. Sleep duration is a matter of concern today because it is related to health $(\mathrm{Ju}$ \& Choi, 2013).

The decrease in sleep duration and quality is currently an endemic problem (Van Cauter, et al., 2008). Sleep duration that was too short or too long was associated with health complications. Studies suggest that waist circumference that is more than normal was associated with less and more sleep duration. Sleep duration> 10 hours/ day was associated with waist circumference and abnormalities of fasting cholesterol, triglyceride, and blood glucose levels (Kim, et al., 2018). The risk of suffering from metabolic syndrome was greater in adolescents with less sleep duration (Hemati, et al., 2018).

The effect of sleep duration on health has been widely studied in adult subjects, such as the incidence of cardiovascular disease (Magee, et al., 2012). Insufficient sleep duration also increased mortality in cardiovascular disease, diabetes, hypertension, and obesity (Itani et al., 2017). 
Research on sleep duration with metabolic syndrome in obese adolescents is still limited. Previous studies on sleep duration have categorized different hours/days of sleep duration. Studies in adolescents have shown an association between sleep duration and metabolic syndrome (Hemati, et al., 2018), but other studies have shown no association (Sung, et al., 2011). This study aimed to analyze the differences in sleep duration between obese adolescents who suffer from metabolic syndrome or not.

\section{METHODS}

This cross sectional study was conducted on obese adolescents who visited the Children's Nutrition and Metabolic Disease Clinic Dr. Soetomo, Surabaya. Subjects aged 13-16 years were taken using a total sampling technique that met the inclusion and exclusion criteria. The inclusion criteria in this study included adolescents aged 13-16 years, able to understand Indonesian, and having parents who signed their readiness to participate in the study. Obese adolescents who suffer from secondary obesity, suffer from endocrine disorders, receive hormonal therapy, smoke, and consume alcohol were excluded in this study. A total of 59 obese adolescents visited the Children's Nutrition and Metabolic Disease Clinic Dr. Soetomo in August - November 2018 who met the inclusion and exclusion criteria were recruited into the study.

Obesity was classified based on CDC 2000. Subjects were obese if the BMI for Age (BMI/A) was above 95th percentile according to age and sex. Subjects suffer from metabolic syndrome if the criteria for metabolic syndrome were obtained according to the International Diabetes Federation (2007) including waist circumference $\geq 90$ percentiles for age and gender accompanied by 2 of the following criteria: (a) fasting triglyceride levels $\geq 150 \mathrm{mg} / \mathrm{dL}$, (b) cholesterol levels Fasting HDL $<40 \mathrm{mg} / \mathrm{dL}$, (c) systolic or diastolic blood pressure $\geq 90$ percentile, and (d) fasting blood glucose levels $\geq 100 \mathrm{mg} / \mathrm{dL}$ (Zimmet et al., 2007).

Sleep duration data were obtained through direct interviews. Subjects were asked to report their sleep and waking hours every day for the past three days. Sleep duration is less if the sleep duration is $<8$ hours a day (Garaulet, et al., 2011a).

Physical examination of the subjects consisted of anthropometric measurements and blood pressure checks. Anthropometric measurements carried out on subjects included body weight, height and waist circumference. Measurement of body weight was carried out using a digital scale scale (Seca, Germany No ref. 224 1714009) with an accuracy of $0.1 \mathrm{~kg}$. Height measurements are carried out using a stadiometer (Seca, Germany No ref. 224 1714009). Height is measured in $\mathrm{cm}$ with an accuracy of $0.1 \mathrm{~cm}$. Waist circumference was measured to determine central obesity using metlin (OneMed, Indonesia) which has an accuracy of $0.1 \mathrm{~cm}$ from the midpoint between the apex of the superior iliac crest and the last rib at the end of expiration expressed in units of $\mathrm{cm}$. Subjects suffered from central obesity if a waist circumference was $\geq 90$ percentile according to age and sex.

Blood pressure checks were performed using a digital blood pressure measuring device (OneMed, Indonesia). Blood pressure checks were performed on the subject in a sitting position after the subject had rested for 10 minutes.

Blood sampling was conducted at 08.00 09.00 am after the subjects had fasted for 12 hours through the median cubital vein. Ten cc of blood was drawn. HDL cholesterol examination was carried out with the HDL-Cholesterol Kit, namely Cholestest ${ }^{\circledR}$ HDL (Sekisui Medical Co., Ltd., Japan) using the TMS Premium 24i tool. Triglyceride examination was carried out with Autosera S TG-N (Sekisui Medical Co., Ltd., Japan) using the TMS Premium 24i tool. Checking fasting blood glucose levels was carried out using glucose hexokinase reagent FS (DiaSys Diagnostic System, Germany) using the TMS Premium 24i tool.

Sleep duration in subjects was described as means and standard deviation. Differences in sleep duration among obese adolescents with or without metabolic syndrome was analyzed by Chi square test using SPSS version 21.0. The study was conducted after obtaining permission from the ethics committee at RSUD Dr. Soetomo, Surabaya (No. 0411/KEPK/VII/2018). Prior to the study, parents were explained about the study to obtain 
informed consent. After the informed consent is signed, the research is carried out on the subject.

\section{RESULT AND DISCUSSION}

In this study, 59 subjects aged 13-16 years were found. A total of $45.8 \%$ had metabolic syndrome and $54.2 \%$ had no metabolic syndrome (Table 1). Most of the obese adolescents (96.6\%) suffer from central obesity. A total of $22.1 \%$ of subjects had triglyceride levels $\geq 150 \mathrm{mg} / \mathrm{dL}$. A total of $32.2 \%$ of subjects had HDL cholesterol levels $<40 \mathrm{mg} / \mathrm{dL}$. As many as $59.3 \%$ of subjects had systolic or diastolic blood pressure $\geq 90$ percentile, and $3.4 \%$ of subjects had fasting blood glucose levels $\geq 100 \mathrm{mg} / \mathrm{dL}$. Subjects suffering from obesity based on BMI/A above 95 percentile according to age and sex.

Metabolic syndrome criteria according to the International Diabetes Federation (2007) include waist circumference $\geq 90$ percentile for age and sex accompanied by 2 of the following criteria: (a) fasting triglyceride levels $\geq 150 \mathrm{mg} / \mathrm{dL}$, (b) fasting HDL cholesterol levels $<40 \mathrm{mg} / \mathrm{dL}$, (c) systolic or diastolic blood pressure $\geq 90$ th percentile, and (d) fasting blood glucose level $\geq 100 \mathrm{mg} / \mathrm{dL}$ (Zimmet, et al., 2007).

The sleep duration of obese adolescents with metabolic syndrome was almost the same as those of obese adolescents who did not suffer from metabolic syndrome (<8 hours/day). Most (57.6\%)

Table 1. Characteristics of Research Subjects

\begin{tabular}{lrrrr}
\hline \multicolumn{1}{c}{ Variable } & \multicolumn{2}{c}{$\begin{array}{c}\text { Metabolic } \\
\text { Syndrome }\end{array}$} & \multicolumn{2}{c}{$\begin{array}{c}\text { Non Metabolic } \\
\text { Syndrome }\end{array}$} \\
\cline { 2 - 6 } & $\mathbf{n}$ & $\mathbf{\%}$ & $\mathbf{n}$ & $\mathbf{\%}$ \\
\hline $\begin{array}{l}\text { Gender } \\
\text { Male }\end{array}$ & 20 & 33.9 & 12 & 20.3 \\
$\quad \begin{array}{l}\text { Female } \\
\text { Metabolic Syndrome Criteria }\end{array}$ & & & & \\
$\quad \begin{array}{l}\text { Waist circumference } \\
\geq 90 \text { percentile }\end{array}$ & 27 & 45.8 & 30 & 50.8 \\
$\begin{array}{l}\text { Triglyceride } \geq 150 \\
\text { mg/dL }\end{array}$ & 8 & 13.6 & 5 & 8.5 \\
$\begin{array}{l}\text { HDL }<40 \text { mg/dL } \\
\text { Systolic or diastolic }\end{array}$ & 15 & 25.4 & 4 & 6.8 \\
$\geq 90$ persentil & 24 & 40.7 & 11 & 18.6 \\
$\begin{array}{l}\text { Fasting blood } \\
\text { glucose } \geq 100 \mathrm{mg} / \mathrm{dL}\end{array}$ & 2 & 3.4 & 0 & 0 \\
\hline
\end{tabular}

Table 2. Differences in Sleep Duration in Obesity Subjects

\begin{tabular}{lccccc}
\hline \multirow{2}{*}{ Sleep Duration } & \multicolumn{2}{c}{$\begin{array}{c}\text { Metabolic } \\
\text { Syndrome }\end{array}$} & \multicolumn{2}{c}{ Non Metabolic } \\
& \multicolumn{2}{c}{ Syndrome } & \multirow{2}{*}{$\mathbf{p}$} \\
\cline { 2 - 5 } & $\mathbf{n}$ & $\mathbf{\%}$ & $\mathbf{n}$ & $\mathbf{\%}$ & \\
\hline$<8$ hours/day & 10 & 16.9 & 15 & 25.4 & \multirow{2}{*}{0.45} \\
$\geq 8$ hours/day & 17 & 28.8 & 17 & 28.8 & \\
\hline
\end{tabular}

obese adolescents had adequate sleep duration, namely $\geq 8$ hours/day.

There was no difference in sleep duration between obese adolescents with metabolic syndrome and those without metabolic syndrome $(p=0.446)$. Less sleep duration interferes with appetite regulation and glucose metabolism thereby increasing the risk of obesity (Van Cauter et al., 2008). Increased ghrelin and decreased leptin occur in individuals who have less sleep duration, thus increasing hunger and associated with increased consumption of high-calorie foods (Spiegel et al., 2004). Chronic lack of sleep duration affects adiposity in children which is associated with metabolic risk (Cespedes et al., 2014).

Metabolic syndrome is associated with poor sleep duration. Adolescents who slept $<8$ hours / day performed longer sedentary activities, such as watching television and consuming less fruit and vegetables than adolescents who had sleep duration $\geq 8$ hours a day (Garaulet et al., 2011a). In this study, there was no difference in sleep duration between obese adolescents with or without metabolic syndrome (Table 2). This was in accordance with previous studies in obese adolescents, which states that sleep duration was not related to metabolic syndrome (Sung et al., 2011; Lee \& Park, 2014).

Another study stated that the relationship between metabolic syndrome and sleep duration depended on the dose or duration of sleep (Iftikhar et al., 2015). Ethnic differences in subjects can have different effects on sleep duration and metabolic syndrome. Previous studies explaining the relationship between sleep duration and metabolic syndrome were mostly carried out in adolescents in European countries (Garaulet, et al., 2011b). Studies in Korea explained that there was no difference in metabolic syndrome in adolescents based on sleep duration (Seo \& Shim, 2019). 
This study had several limitations. Sleep duration was assessed based on the subject's perception, so that recall bias could occur. This study did not assess the duration of sleep on the subjects' school days and weekends so that the total sleep duration in one week in the subjects could be different. Blood examination of the subjects was carried out once. Serial blood tests are required to reduce the influence of the circadian rhythm and the random fluctuations of increasing or decreasing levels of fat or blood glucose.

\section{CONCLUSION}

There was no difference in sleep duration between obese adolescents with metabolic syndrome compared with obese adolescents without metabolic syndrome. Assessment of sleep duration at the subjects' school days and weekends in obese adolescents is needed to assess the association between sleep duration and the risk of metabolic syndrome.

\section{REFERENCES}

Azadbakht, L., Kelishadi, R., Khodarahmi, M., Qorbani, M., Heshmat, R., Motlagh, M.E., Taslimi, M., \& Ardalan, G. (2013). The association of sleep duration and cardiometabolic risk factors in a national sample of children and adolescents: The CASPIAN III Study. Nutrition, 29(9), 1133-1141. doi: 10.1016/ j.nut.2013.03.006.

Börnhorst, C., Hense, S., Ahrens, W., Hebestreit, A., Reisch, L., Barba, G., von Kries, R., Bayer, O., \& IDEFICS Consortium. (2012). From sleep duration to childhood obesity - What are the pathways? European Journal of Pediatrics, 171(7), 1029-1038. doi: 10.1007/ s00431-0111670-8.

Cespedes, E.M., Rifas-Shiman, S.L., Redline, S., Gillman, M.W., Peña, M.M., \& Taveras, E.M. (2014). Longitudinal associations of sleep curtailment with metabolic risk in midchildhood. Obesity (Silver Spring, Md.), 22(12), 2586-2592. doi: 10.1002/oby.20894.

Garaulet, M., Ortega, F.B., Ruiz, J.R., Rey-López, J.P., Béghin, L., ... Moreno, L.A. (2011). Short sleep duration is associated with increased obesity markers in European adolescents: Effect of physical activity and dietary habits.
The HELENA study. International Journal of Obesity, 35(10), 1308-1317. doi: 10.1038/ ijo.2011.149.

Guo, X., Zheng, L., Li, Y., Yu, S., Liu, S., Zhou, X.,... Sun, Y. (2011). Association between sleep duration and hypertension among Chinese children and adolescents. Clinical Cardiology, 34(12), 774-781. doi: 10.1002/clc.20976.

Hemati, Z., Mozafarian, N., Heshmat, R., Ahadi, Z., Motlagh, M. E., Ziaodini, H., ... Kelishadi, R. (2018). Association of sleep duration with metabolic syndrome and its components in children and adolescents; a propensity scorematched analysis: The CASPIAN-V study. Diabetology \& Metabolic Syndrome, 10(1). doi: 10.1186/s13098-018-0381-y.

Iftikhar, I.H., Donley, M.A., Mindel, J., Pleister, A., Soriano, S., \& Magalang, U.J. (2015). Sleep Duration and Metabolic Syndrome. An Updated Dose-Risk Metaanalysis. Annals of the American Thoracic Society, 12(9), 1364-1372. doi: 10.1513/AnnalsATS.201504-190OC.

Itani, O., Jike, M., Watanabe, N., \& Kaneita, Y. (2017). Short sleep duration and health outcomes: A systematic review, meta-analysis, and meta-regression. Sleep Medicine, 32, 246256. doi: 10.1016/j.sleep.2016.08.006.

Ju, S.Y. \& Choi, W.S. (2013). Sleep duration and metabolic syndrome in adult populations: A meta-analysis of observational studies. Nutrition \& Diabetes, 3(5), e65-e65. doi: 10.1038/ nutd.2013.8.

Kim, C.E., Shin, S., Lee, H.W., Lim, J., Lee, J., Shin, A., \& Kang, D. (2018). Association between sleep duration and metabolic syndrome: A crosssectional study. BMC Public Health, 18(1). doi: 10.1186/s12889-018-5557-8.

Kong, A.P., Wing, Y.K., Choi, K.C., Li, A.M., Ko, G.T.C., Ma, R.C., ... Chan, J.C. (2011). Associations of sleep duration with obesity and serum lipid profile in children and adolescents. Sleep Medicine, 12(7), 659-665. doi: 10.1016/j. sleep.2010.12.015.

Lee, J.A. \& Park, H.S. (2014). Relation between sleep duration, overweight, and metabolic syndrome in Korean adolescents. Nutrition, Metabolism and Cardiovascular Diseases, 24(1), 65-71. doi: 10.1016/j.numecd.2013.06.004.

Magee, C.A., Kritharides, L., Attia, J., Mcelduff, P., $\&$ Banks, E. (2012). Short and long sleep duration are associated with prevalent cardiovascular disease in Australian adults: Sleep duration 
and cardiovascular disease. Journal of Sleep Research, 21(4), 441-447. doi: 10.1111/j.13652869.2011.00993.x.

Rochlani, Y., Pothineni, N.V., Kovelamudi, S., \& Mehta, J.L. (2017). Metabolic syndrome: Pathophysiology, management, and modulation by natural compounds. Therapeutic Advances in Cardiovascular Disease, 11(8), 215-225. doi: $10.1177 / 1753944717711379$.

Seo, S.H. \& Shim, Y.S. (2019). Association of sleep duration with obesity and cardiometabolic risk factors in children and adolescents: a population-based study. Scientific Reports, 9(1). doi: 10.1038/s41598-019-45951-0.

Spiegel, K., Tasali, E., Penev, P., \& Cauter, E.V. (2004). Brief Communication: Sleep Curtailment in Healthy Young Men Is Associated with Decreased Leptin Levels, Elevated Ghrelin Levels, and Increased Hunger and Appetite. Annals of Internal Medicine, 141(11), 846. doi: 10.7326/0003-4819-141-11-20041207000008
Sung, V., Beebe, D.W., Vandyke, R., Fenchel, M.C., Crimmins, N.A., Kirk, S., Harriet, H., Raouf, Amin., Wake, M. (2011). Does sleep duration predict metabolic risk in obese adolescents attending tertiary services? A cross-sectional study. Sleep, 34(7), 891-898. doi: 10.5665/ SLEEP.1122.

Van Cauter, E., Spiegel, K., Tasali, E., \& Leproult, R. (2008). Metabolic consequences of sleep and sleep loss. Sleep Medicine, 9, S23-S28. doi: 10.1016/S1389-9457(08)70013-3.

Vgontzas, A.N., Zoumakis, E., Bixler, E.O., Lin, H.M., Follett, H., Kales, A., \& Chrousos, G.P. (2004). Adverse Effects of Modest Sleep Restriction on Sleepiness, Performance, and Inflammatory Cytokines. The Journal of Clinical Endocrinology \& Metabolism, 89(5), 2119-2126. do: i10.1210/ jc.2003-031562.

Zimmet, P., Alberti, K.G.M., Kaufman, F., Tajima, N., Silink, M., Arslanian, S., Wong, G., Bennett, P., Shaw, J., Caprio, S., \& IDF Consensus Group. (2007). The metabolic syndrome in children and adolescents? An IDF consensus report. Pediatric Diabetes, 8(5), 299-306. doi: 10.1111/j.1399-5448.2007.00271.x. 